\title{
Disparity of perspectives between teachers and learners on perioperative teaching and learning
}

\author{
Yu-Tang Chang ${ }^{1,2}$, Peih-Ying $L u^{2,3}$ and Chung-Sheng Lai ${ }^{1,2^{*}}$
}

\begin{abstract}
Background: To build a consensus about learning objectives in the operating room, the aim of the study was to evaluate both surgical teacher and learner perspectives on perioperative teaching and learning in Taiwan.

Methods: Twelve main technical and non-technical learning objectives in the operating room were evaluated by learners and surgical teachers in Kaohsiung Medical University Hospital. The learners included postgraduate year (PGY) 1-3 residents (junior learner, JL) and PGY 4-7 residents (senior learner, SL). The definition of learning preferences were recommended learning objectives, and learning load was defined as demands of learning preferences. During the survey, surgical teachers evaluated the learning preferences for the learner, and learners evaluated their learning preferences. The learners also evaluated the learning preferences that the surgical teachers should teach.
\end{abstract}

Results: Response rate of the questionnaire was $65.4 \%$. A total of 31 learners and 39 surgical teachers completed the survey. The consensus was that the need to increase the learning loads and ethical issues were the learning preferences for SL, and indications, details of procedure, and teamwork were important to both JL and SL. The teachers intended to set specific learning objectives for different learner levels, including (i) indications, details of procedure, teamwork, and postoperative care for both $J \mathrm{~L}$ and $\mathrm{SL}$; (ii) preoperative preparation, surgical anatomy, and instrument handling for $J \mathrm{~L}(P=0.022,0.021$ and 0.006); and (iii) surgical technique, independent practice, clinical reasoning, complications, and ethical issues for $S L(P=0.010,<0.001,<0.001,0.001,0.011)$. Resident perspective on learning objectives differed between $J \mathrm{~L}$ and $\mathrm{SL}$, and there was discrepancy between resident's learning as perceived by teachers, particularly in the $\mathrm{J}$.

Conclusions: Our study revealed significant disparity of perspectives between teachers and learners on perioperative teaching and learning. Surgical teachers should set specific learning objectives for different learner levels, since junior and senior residents have different learning preferences even though both scrub in the same case. Effective communication between teachers and learners has the potential to improve learning experience and create a positive environment in the operating room.

Keywords: Perioperative teaching and learning, Operating room, Perspectives, Learning objectives, Working hours

\footnotetext{
* Correspondence: chshla@kmu.edu.tw

'Department of Surgery, Kaohsiung Medical University Hospital, Kaohsiung,

Taiwan

${ }^{2}$ School of Medicine, College of Medicine, Kaohsiung Medical University,

Kaohsiung, Taiwan

Full list of author information is available at the end of the article
}

(C) The Author(s). 2020 Open Access This article is licensed under a Creative Commons Attribution 4.0 International License, which permits use, sharing, adaptation, distribution and reproduction in any medium or format, as long as you give appropriate credit to the original author(s) and the source, provide a link to the Creative Commons licence, and indicate if changes were made. The images or other third party material in this article are included in the article's Creative Commons licence, unless indicated otherwise in a credit line to the material. If material is not included in the article's Creative Commons licence and your intended use is not permitted by statutory regulation or exceeds the permitted use, you will need to obtain permission directly from the copyright holder. To view a copy of this licence, visit http://creativecommons.org/licenses/by/4.0/ The Creative Commons Public Domain Dedication waiver (http://creativecommons.org/publicdomain/zero/1.0/) applies to the data made available in this article, unless otherwise stated in a credit line to the data. 


\section{Background}

The apprenticeship model for surgical training has been a long-standing gold standard worldwide, with significant time demands of learners working under supervision until being judged competent to operate on their own $[1,2]$. However, long working hours might increase the risk of making medical errors [3]. Therefore, duty hours of the residents in the United States have been restricted by the Accreditation Council for Graduate Medical Education Common Program Requirements since 2003 [4]; similar standards were not implemented in Taiwan until 2013. In March 2017, the Ministry of Health and Welfare of Taiwan announced working-hour guidelines for resident physicians where maximum weekly working hours were reduced from 88 to 80 [5]. Starting from September 2019, residents were incorporated into the National Labor Standards Act. At present, residents in Taiwan have fixed work hours and work under a responsibility system [6]. With more restrictions on working hours, the working enviro nment for resident physicians and patient safety can be improved [4]; however, due to limited time in the hospital, it is necessary to improve the efficiency of surgical training for resident physicians [7-9].

The importance of intraoperative teaching is coming into great focus $[2,10-14]$. Several interventions and principles of practice have been developed to improve intraoperative education $[7,12,15,16]$. The briefing, intraoperative teaching, debriefing "BID" model reported by Roberts et al. identified that the teacher should "brief" the learner before the case, then teach in the operating room, and then "debrief" about what goals were met and how learners can improve for the next case [15]; however, there is significant discrepancy between resident and surgical teacher perspectives on preoperative preparation as well as intraoperative teaching and postoperative feedback [17-20]. To improve the learning environment in the operating room, the learners and the teachers both need to build a consensus about learning and teaching objectives. In Taiwan, few studies have demonstrated the resident and surgical teacher perspectives in the operating room [8]; accordingly, the purpose of this study was to evaluate learner and teacher perspectives on learning and teaching objectives in the operating room.

\section{Methods}

\section{Study setting}

In Taiwan, the residency training program has followed the similar program of the American Medical Association since 1950 [21]. Medical students graduate from medical school after completing 6 years of college education and 1 year of internship training and then chose their specialty in the residency training program. In
2003, a 3-month post-graduation general medicine training program was implemented throughout Taiwan, and 1 year of postgraduate general medicine training was introduced [21]. Since 2013, the medical education program has changed from 7 years to 6 years, moving the last year internship to postgraduate year (PGY) 1 resident that is like the training systems in Japan and in the United Kingdom [22, 23].

The Institutional Review Board at Kaohsiung Medical University Hospital approved this study (KMUHIRB-E(II)-20,180,262). Participation in the survey was voluntary and without compensation. Teachers were attending surgeons from various surgical specialties, whereas learners included PGY 1-3 residents (junior learner, JL), and PGY 4-7 residents (senior learner, SL) [19]. Participant surgeons and learners provided written informed consent and completed the survey.

\section{Survey instrument}

We have referred to the resident performance assessments in the American Broad Surgery (ABS) and Non-Technical Skills for Surgeons (NOTSS) behavior marker system defined by the Royal College of Surgeons of Edinburgh when creating our survey instrument $[24,25]$. The assessment of operative and clinical performance in the ABS standardizes the knowledge and skills (e.g. steps of procedure and independent practice) expected of general surgery residents, while the NOTSS behavior marker system was developed to evaluate learner non-technical performance. Then we had a panel of experts reach consensus regarding what technical and non-technical skills to be included. We also reviewed related literature to construct detailed learning objectives. Agha et al. has emphasized that non-technical skills such as communication and teamwork skills and decision-making are of increasing importance in surgery and surgical training [9]. Rose et al. reported that technical skills such as operative steps, surgical skills, instrument handling, and surgical technique were significantly different between surgical residents and faculty [17]. Furthermore, local context was considered, and Lai et al. have reported that teaching of clinical reasoning in surgery is of paramount importance to stimulate learner's self-directed learning $[8,26]$. To evaluate the complete educational activities in the operating room, learning objectives before and after surgery were also included. Therefore, 12 specific technical and nontechnical learning objectives, including preoperative preparation, surgical anatomy, operative indications, instrument handling, surgical technique, details of procedure, independent practice, clinical reasoning, complications, teamwork, ethical issues and 
postoperative care were evaluated using a standardized questionnaire.

The definition of perioperative teaching was expected objectives provided by surgical teachers, while the definition of perioperative learning was objectives that residents desired to learn. Among twelve main technical and non-technical learning objectives, preoperative preparation was defined as the preparation before the surgery in the operating room such as aseptic environment, temperature and patient position. Details of procedure referred to explicit explanation and verbally describing surgical procedures step-by-step. Ethical issues in surgery were defined as medical errors, truth-telling, and disclosure [27]. Postoperative care was defined as the care after surgery in the operating room such as evaluation of skin integrity, patient transportation and immediate operative reports. The definition of learning preference included technical and non-technical skills that were recommended to be learnt, and learning load was defined as demands of learning preferences.

\section{Data collection}

During the survey, surgical teachers evaluated the learning preferences for the learner (Group I, teacher perspective on learning), and learners evaluated their learning preferences (Group II, learner perspective on learning). The learners also evaluated the learning preferences that the surgical teachers should teach (Group III, learner perspective on teaching). More detailed information on the performance characteristics of the survey instruments, including the original Chinese-language survey, and English-language version, are provided in the Additional file 1.

Surgical teachers were analyzed for their demographic variables (age at evaluation, gender, seniority as an attending surgeon, seniority as a faculty member of the medical school, experience of annual teaching award, and percentage of effort teaching). Secondly, 12 selected objectives were evaluated among the groups. The perspectives between junior and senior learners were also compared.

\section{Statistical analysis}

Student $t$-tests and $\chi^{2}$ tests were used to compare continuous and categorical descriptive variables respectively between learners and surgical teachers. The differences in enrolled learners and surgical teachers were compared across three groups using analysis of variance (ANOVA) for continuous variables and $\chi^{2}$ tests for categorical variables. Further analysis with Scheffé post-hoc test was planned when the ANOVA showed that there was a statistical difference between groups. A $P$ value less than 0.05 denoted statistical significance. SPSS for Windows version 20.0 was used for all statistics.

\section{Results}

\section{Demographic data}

In September 2018, a total of 65 surgical teachers and 42 learners were surveyed. We collected responses from 31 learners and 39 surgical teachers (response rate: 65.4\%). The 31 learners included two PGY1 residents, 11 PGY2 residents, six PGY3 residents, three PGY4 residents, five PGY5 residents, three PGY6 residents and one PGY7 resident. Overall, there were $19 \mathrm{JL}$ and $12 \mathrm{SL}$.

The characteristics of participating surgical teachers are shown in Table 1 . The average seniority as an attending surgeon was $14.9 \pm 10.9$ years, ranging from 1 to 40 years. Of 21 surgeons who had faculty positions at the medical school, one was a lecturer, eight were assistant professors, four were associate professors, and eight were full professors. Most of the teachers (56.4\%) responded that teaching constituted between 21 and $40 \%$ of their overall efforts. Eighteen surgeons indicated they had received at least one teaching award during their career. Among 39 surgical teachers, there were 29 (74.4\%) general surgeons, 4 (10.2\%) plastic surgeons and $6(15.4 \%)$ neurosurgeons. There were no statistically significant differences in age, seniority as an attending surgeon, seniority as a faculty member of the medical school, and experience of annual teaching award among three specialties $(P=0.357,0.453,0.803$ and 0.415$)$.

\section{Comparison between junior learners and senior learners Teacher perspective on learning (group I)}

The surgical teachers perceived that SL should require more learning objectives $(8.9 \pm 3.0$ vs $7.8 \pm 2.5 ; \quad P=$ $0.049)$. Instrument handling $(84.6 \%)$ was the most important learning objective for $\mathrm{JL}$, while independent practice $(94.9 \%)$ was indicated for SL. In comparison between JL and SL, preoperative preparation, surgical anatomy and instrument handling were indicated as preferences for JL $(P=0.022,0.021$, and 0.006), while surgical technique, independent practice, clinical

Table 1 Characteristics of surgical teachers

\begin{tabular}{llll}
\hline Surgical teachers $(N=39)$ & Items & $N$ & $\%$ \\
\hline Gender & Male & 36 & 92.3 \\
& Female & 3 & 7.7 \\
Age & $31-50$ years & 23 & 59.0 \\
& $>50$ years & 16 & 41.0 \\
Seniority as an attending & $<15$ years & 19 & 48.7 \\
& $\geq 15$ years & 20 & 51.3 \\
Faculty member of medical school & No & 18 & 46.2 \\
& Yes & 21 & 53.8 \\
Effort teaching (\%) & $0-20 \%$ & 8 & 20.5 \\
& $21-40 \%$ & 22 & 56.4 \\
& $>41 \%$ & 9 & 23.1 \\
\hline
\end{tabular}


reasoning, complications and ethical issues were indicated for SL $(P=0.010,<0.001,<0.001,0.001$, and $0.011)$. Details of procedure and teamwork were recommended learning preferences for both $\mathrm{JL}$ and SL (Table 2).

\section{Learner perspective on learning (group II)}

$\mathrm{JL}$ perceived that surgical technique (84.2\%) was the most important learning objective; meanwhile, both surgical anatomy and indications (91.7\%) were indicated for SL. In comparison between JL and SL, ethical issues and postoperative care were the SL's learning preferences $(P=0.027$ and 0.038$)$ (Table 2).

\section{Learner perspective on teaching (group III)}

The learners perceived that surgical teachers should teach more objectives to SL $(6.8 \pm 4.1$ vs $4.4 \pm 2.4 ; P=$ $0.007)$. JL perceived that surgical anatomy $(63.2 \%)$ and indications $(63.2 \%)$ were the learning objectives that surgical teacher should provide; meanwhile, SL perceived that surgical anatomy (75\%), surgical technique (75\%) and independent practice (75\%) were the objectives. In comparison between JL and SL, independent practice and ethical issues were the SL's preferences that the teachers should teach $(P=0.018$ and 0.014$)$ (Table 2).

\section{Surgical teacher and learners perspectives among the three groups}

The teachers, JL and SL shared common ground when discussing indications, details of procedure, teamwork, and ethical issues, with the consensus being that ethical issues were the learning preference for $\mathrm{SL}$, whereas indications, details of procedure, and teamwork were important to both JL and SL. However, resident perspective on learning objectives differed between JL and SL, and there was discrepancy between resident's learning as perceived by teachers, particularly in the JL (Table 2).

\section{Comparison of surgical teacher and learner perspectives on learning (group I vs. group II)}

The demands of the learning objectives between surgical teacher and learner preferences were compared. For JL, the number of the learning objectives from the surgical teacher perspective was more than those from the learner perspective $(P=0.042)$; however, there was no significant difference for SL. Of the 12 objectives, JL responses differed from teacher responses on five objectives and SL responses on three. From teacher perspective, preoperative preparation, instrument handling, teamwork and postoperative care were the preferences for the JL $(P=0.019,<0.001,0.020$ and 0.031$)$; however, the JL' s preference was independent practice $(P=0.007)$. SL saw surgical anatomy was the learning preference $(P=0.005)$; whereas clinical reasoning and complications were the preferences for SL from the teacher perspective $(P=0.001$, and 0.024$)$ (Table 3$)$.

Comparison between teacher perspective on learning and learner perspective on teaching (group I vs. group III) Learner and teacher perspectives on learning loads were compared. The surgical teacher perceived that JL

Table 2 Teacher and learner perspectives in the operating room

\begin{tabular}{|c|c|c|c|c|c|c|c|c|c|}
\hline \multirow[t]{2}{*}{ Learning objectives } & \multicolumn{2}{|c|}{$\begin{array}{l}\text { Group I } \\
\text { Teacher perspective on } \\
\text { learning }\end{array}$} & \multirow[t]{2}{*}{ P } & \multicolumn{2}{|c|}{$\begin{array}{l}\text { Group II } \\
\text { Learner perspective on } \\
\text { learning }\end{array}$} & \multirow[t]{2}{*}{$P$} & \multicolumn{2}{|c|}{$\begin{array}{l}\text { Group III } \\
\text { Learner perspective on } \\
\text { teaching }\end{array}$} & \multirow[t]{2}{*}{$P$} \\
\hline & $\begin{array}{l}\mathrm{J} \\
\mathrm{N}(\%)\end{array}$ & $\begin{array}{l}\mathrm{SL} \\
\mathrm{N}(\%)\end{array}$ & & $\begin{array}{l}\mathrm{JL} \\
\mathrm{N}(\%)\end{array}$ & $\begin{array}{l}\mathrm{SL} \\
\mathrm{N}(\%)\end{array}$ & & $\begin{array}{l}\mathrm{J} \\
\mathrm{N}(\%)\end{array}$ & $\begin{array}{l}\mathrm{SL} \\
\mathrm{N}(\%)\end{array}$ & \\
\hline Number of learning objectives & $7.8 \pm 2.5$ & $8.9 \pm 3.0$ & 0.049 & $6.3 \pm 3.5$ & $8.3 \pm 3.5$ & 0.795 & $4.4 \pm 2.4$ & $6.8 \pm 4.1$ & 0.007 \\
\hline Preoperative preparation & $27(69.2)$ & $17(43.6)$ & 0.022 & $7(36.8)$ & $7(58.3)$ & 0.242 & $6(31.6)$ & $6(50.0)$ & 0.305 \\
\hline Surgical anatomy & $28(71.8)$ & $18(46.2)$ & 0.021 & $14(73.7)$ & $11(91.7)$ & 0.132 & $12(63.2)$ & $9(75.0)$ & 0.492 \\
\hline Indications & $32(82.1)$ & $25(64.1)$ & 0.074 & $13(68.4)$ & $11(91.7)$ & 0.217 & $12(63.2)$ & $8(66.7)$ & 0.842 \\
\hline Instrument handling & $33(84.6)$ & $22(56.4)$ & 0.006 & $7(36.8)$ & $6(50.0)$ & 0.470 & $2(10.5)$ & $4(33.3)$ & 0.117 \\
\hline Surgical technique & $27(69.2)$ & $36(92.3)$ & 0.010 & $16(84.2)$ & $10(83.3)$ & 0.948 & $10(52.6)$ & $9(75.0)$ & 0.213 \\
\hline Details of procedure & $32(82.1)$ & $32(82.1)$ & 1.000 & $14(73.7)$ & $8(66.7)$ & 0.675 & $9(47.4)$ & $6(50.0)$ & 0.886 \\
\hline Independent practice & $12(30.8)$ & $37(94.9)$ & $<0.001$ & $13(68.4)$ & $10(83.3)$ & 0.355 & $6(31.6)$ & $9(75.0)$ & 0.018 \\
\hline Clinical reasoning & $18(46.2)$ & $36(92.3)$ & $<0.001$ & $9(47.4)$ & $6(50.0)$ & 0.886 & $6(31.6)$ & $6(50.0)$ & 0.305 \\
\hline Complications & 24 (61.6) & $36(92.3)$ & 0.001 & $9(47.4)$ & $8(66.7)$ & 0.293 & 6 (31.6) & $8(66.7)$ & 0.056 \\
\hline Teamwork & $25(64.1)$ & $28(71.8)$ & 0.467 & $6(31.6)$ & $5(38.5)$ & 0.567 & $5(26.3)$ & $5(35.8)$ & 0.373 \\
\hline Ethical issues & $18(46.2)$ & $29(74.4)$ & 0.011 & $5(26.3)$ & $8(66.7)$ & 0.027 & $2(10.5)$ & $6(50.0)$ & 0.014 \\
\hline Postoperative care & $26(66.7)$ & $29(74.4)$ & 0.456 & $7(36.8)$ & $9(75.0)$ & 0.038 & $8(42.1)$ & $6(50.0)$ & 0.667 \\
\hline
\end{tabular}


Table 3 Comparison of learner and teacher perspectives on learning (Group I vs. Group II) in the operating room

\begin{tabular}{|c|c|c|c|c|c|c|}
\hline \multirow[t]{3}{*}{ Learning objectives } & \multicolumn{2}{|l|}{ Junior learner } & \multirow[t]{3}{*}{$P$} & \multicolumn{2}{|l|}{ Senior learner } & \multirow[t]{3}{*}{$P$} \\
\hline & Learner perspective & Teacher perspective & & Learner perspective & Teacher perspective & \\
\hline & $N=19$ & $N=39$ & & $N=12$ & $N=39$ & \\
\hline Number of learning objectives & $6.3 \pm 3.5$ & $7.8 \pm 2.5$ & 0.042 & $8.3 \pm 3.5$ & $8.9 \pm 3.0$ & 0.334 \\
\hline Preoperative preparation & $7(36.8)$ & $27(69.2)$ & 0.019 & $7(58.3)$ & $17(43.6)$ & 0.371 \\
\hline Surgical anatomy & $14(73.7)$ & $28(71.8)$ & 0.880 & $11(91.7)$ & $18(46.2)$ & 0.005 \\
\hline Indications & $13(68.4)$ & $32(82.1)$ & 0.243 & $11(91.7)$ & $25(64.1)$ & 0.067 \\
\hline Instrument handling & $7(36.8)$ & $33(84.6)$ & $<0.001$ & $6(50.0)$ & $22(56.4)$ & 0.696 \\
\hline Surgical technique & $16(84.2)$ & $27(69.2)$ & 0.221 & $10(83.3)$ & $36(92.3)$ & 0.361 \\
\hline Details of procedure & $14(73.7)$ & $32(82.1)$ & 0.460 & $8(66.7)$ & $32(82.1)$ & 0.257 \\
\hline Independent practice & $13(68.4)$ & $12(30.8)$ & 0.007 & $10(83.3)$ & $37(94.9)$ & 0.194 \\
\hline Clinical reasoning & $9(47.4)$ & $18(46.2)$ & 0.931 & $6(50.0)$ & 36 (92.3) & 0.001 \\
\hline Complications & $9(47.4)$ & $24(61.6)$ & 0.306 & $8(66.7)$ & $36(92.3)$ & 0.024 \\
\hline Teamwork & $6(31.6)$ & $25(64.1)$ & 0.020 & $5(38.5)$ & 28 (71.8) & 0.056 \\
\hline Ethical issues & $5(26.3)$ & $18(46.2)$ & 0.147 & $8(66.7)$ & $29(74.4)$ & 0.602 \\
\hline Postoperative care & $7(36.8)$ & $26(66.7)$ & 0.031 & $9(75.0)$ & $29(74.4)$ & 0.964 \\
\hline
\end{tabular}

required more learning objectives $(7.8 \pm 2.5)$ compared with the JL perspective $(4.4 \pm 2.4)(P<0.001)$, but there was no significant difference for the SL perspective $(P=$ 0.065). Of the 12 objectives, the disparity of perspectives between JL and teachers was six, including preoperative preparation, instrument handing, details of procedure, complications, teamwork and ethical issues $(P=0.007$, < $0.001,0.006,0.032,0.007$ and 0.007). However, SL responses differed from teacher responses on four objectives, including details of procedure, independent practice, clinical reasoning and complications $(P=0.026$, $0.043,0.001$ and 0.024 ).

The surgical teachers perceived that preoperative preparation, instrument handling, details of procedure, complications, teamwork and ethical issues were the preferences for JL $(P=0.007,<0.001,0.006,0.032,0.007$ and 0.007 ), and details of procedure, independent practice, clinical reasoning and complications were the preferences for SL $(P=0.026,0.043,0.001$, and 0.024) (Table 4).

\section{Discussion}

Due to gradually increasing medical disputes and legal liability, the relationship between physicians and patients has become tense [28]. As a result, physicians have started to choose defensive medicine practice, and the brain drain in high-risk specialties, especially for surgeons, has continued unabated [28, 29]. From 2006 through 2018, according to the Taiwan Medical Association, the number of member physicians has increased from 35,936 to 49,019 ( $2.8 \%$ increase per year) and the number of board-certified surgeons from 3027 to 3830 (2.0\% increase per year) [30]. For every 1000 new physicians, only 61 choose surgery as a career although there are more than 300 positions of surgical residency provided for 1500 postgraduates each year in Taiwan. The dwindling interest in surgery and surgical specialties raises concerns that surgical manpower requirements might reach a deficit in coming years [31]. In 2014, the National Health Research Institutes of Taiwan reported that there will be more than 1500 vacancies of surgical specialty in Taiwan after 2022 [32]. Factors that disaffect young doctors in choosing high-risk specialties such as surgery are long duty hours, increased legal liability, and low payments from the National Health Insurance System [32]. The restriction on duty hours is the policy to improve the working environment for resident physicians. However, the teaching hospital is normally concerned that work hour restrictions will limit clinical training time and even worsen the decrease of working hours. In the era of competency-based surgical training, how to create a more effective learning environment has become key to improve surgical education and enhance junior doctors' confidence in pursuing a career in surgery [29].

For the training program of surgical residency, the operating room is the main educational theater. However, our study identified different learning preferences among teachers, junior learners, and senior learners. The educational activity in the operating room cannot be efficient if teachers and learners do not agree on what should be taught. Lack of communication between learners and teachers might account for significant discrepancy between resident and surgical teacher perspectives [2]. Communication barriers in the operating room would impede learning objectives from being efficiently 
Table 4 Comparison between teacher perspective on learning (Group I) and learner perspective on teaching (Group III) in the operating room

\begin{tabular}{|c|c|c|c|c|c|c|}
\hline \multirow[t]{3}{*}{ Learning objectives } & \multicolumn{2}{|l|}{ Junior learner } & \multirow[t]{3}{*}{$P$} & \multicolumn{2}{|l|}{ Senior learner } & \multirow[t]{3}{*}{$P$} \\
\hline & $\begin{array}{l}\text { Teacher perspective on } \\
\text { learning }\end{array}$ & $\begin{array}{l}\text { Learner perspective on } \\
\text { teaching }\end{array}$ & & $\begin{array}{l}\text { Teacher perspective on } \\
\text { learning }\end{array}$ & $\begin{array}{l}\text { Learner perspective on } \\
\text { teaching }\end{array}$ & \\
\hline & $N=39$ & $N=19$ & & $N=39$ & $N=12$ & \\
\hline $\begin{array}{l}\text { Number of learning } \\
\text { objectives }\end{array}$ & $7.8 \pm 2.5$ & $4.4 \pm 2.4$ & $\begin{array}{l}< \\
0.001\end{array}$ & $8.9 \pm 3.0$ & $6.8 \pm 4.1$ & 0.065 \\
\hline $\begin{array}{l}\text { Preoperative } \\
\text { preparation }\end{array}$ & $27(69.2)$ & $6(31.6)$ & 0.007 & $17(43.6)$ & $6(50.0)$ & 0.696 \\
\hline Surgical anatomy & $28(71.8)$ & $12(63.2)$ & 0.505 & $18(46.2)$ & $9(75.0)$ & 0.080 \\
\hline Indications & $32(82.1)$ & $12(63.2)$ & 0.115 & $25(64.1)$ & $8(66.7)$ & 0.871 \\
\hline Instrument handling & $33(84.6)$ & $2(10.5)$ & $\begin{array}{l}< \\
0.001\end{array}$ & $22(56.4)$ & $4(33.3)$ & 0.162 \\
\hline Surgical technique & $27(69.2)$ & $10(52.6)$ & 0.217 & $36(92.3)$ & $9(75.0)$ & 0.104 \\
\hline Details of procedure & $32(82.1)$ & $9(47.4)$ & 0.006 & $32(82.1)$ & $6(50.0)$ & 0.026 \\
\hline Independent practice & $12(30.8)$ & $6(31.6)$ & 0.950 & 37 (94.9) & $9(75.0)$ & 0.043 \\
\hline Clinical reasoning & $18(46.2)$ & $6(31.6)$ & 0.290 & $36(92.3)$ & $6(50.0)$ & 0.001 \\
\hline Complications & $24(61.6)$ & $6(31.6)$ & 0.032 & $36(92.3)$ & $8(66.7)$ & 0.024 \\
\hline Teamwork & $25(64.1)$ & $5(26.3)$ & 0.007 & $28(71.8)$ & $5(35.8)$ & 0.056 \\
\hline Ethical issues & $18(46.2)$ & $2(10.5)$ & 0.007 & $29(74.4)$ & $6(50.0)$ & 0.112 \\
\hline Postoperative care & $26(66.7)$ & $8(42.1)$ & 0.075 & $29(74.4)$ & $6(50.0)$ & 0.112 \\
\hline
\end{tabular}

delivered. However, the relationship involves both sides. For example: teachers may stay silent or keep blaming during the operation, while learners would fear or hesitate to express their expectations. If neither teachers nor learners leverage the communication, it might be difficult for learners to get the most out of their education. The solution to the problem would be for teachers to decide what they will teach at the different learner levels. Effective communication between teachers and learners has the potential to improve the learning experience and create a positive environment in the operating room.

In the past, the volume of surgical procedures was the key to surgical training in the operating room [15]. However, owing to limited duty hours of the residency and the increasing volume of surgical procedures, it is impossible for learners to learn at one time. Unguided (pure) discovery learning is ineffective and inefficient [15]. A good model for deliberate teaching in the operating room would allow the teacher to focus more on setting objectives for the learner's performance, providing immediate and specific feedback, and providing guidance for future practice [15]. Agha et al. recommended that modern surgical practice requires technical and nontechnical skills, evidence-based practice, an emphasis on lifelong learning, monitoring of outcomes, and a supportive institutional and health service framework [9]. Although many aspects of both technical and nontechnical skills development can be achieved outside the operating room, the development and enhancement of certain skills still require learning in the operating room [2], where the types of learning models may be individualistic and determined by the learner's competence and attitudes [10, 17].

Considering perception in the present study, both learners and teachers agreed that the appropriate learning loads for the objectives should increase gradually. However, Rose et al. reported there were significant differences between resident and faculty surgeons with respect to teaching the operative steps, surgical skills, instrument handling and surgical technique [17]. In the present study, in addition to significant disparity in learning and teaching between learner and teacher perceptions, junior and senior residents have different learning needs in the operating room even though both scrub in the same case. Cunha-Melo and Costa reported expectations and competencies of junior learners differ greatly from that of senior learners [19]. Park et al. reported resident perception of confidence differs from junior to senior residents [33]. Torbeck et al. reported more autonomy should be given to senior than to junior residents [34]. Consequently, an expert might better provide guided-discovery learning for the novice with preparatory information before the experience, and offer verbal and perhaps manual guidance when necessary, during the experience and feedback afterward [15].

Technical skills are important for successful surgical practice. Surgical technique was the learning preference from learner perspective; meanwhile, surgical teachers 
perceived that the higher the learner level, the higher priority set on the objectives needed. However, a traditional focus on the acquisition of technical skills and competence is no longer enough for the delivery of modern and safe surgical practice [9]. There is increasing evidence that patient harm is not due to deficient technical skills alone. Poor decision-making and deficiencies in teamwork are reported as contributors to adverse events and patient harm in the operating room. Therefore, non-technical skills are of increasing importance in surgery and surgical training [9]. Several assessment tools have been developed to evaluate learner non-technical performance [35, 36], and the most extensively used and validated training tool is the NOTSS behavior marker system, include situational awareness, decision-making, communication \& teamwork, and leadership [25].

In the present study, learner perspectives on the nontechnical skills such as clinical reasoning and teamwork were significantly different from those of the surgical teacher. The surgical teachers perceived that clinical reasoning was learning preference for SL. Although surgical teachers perceived that successful teamwork was learning preference for both JL and SL, the learners did not regard the learning objective as their main preference. In the operating room, surgeons should need the skill of evidence-based clinical reasoning to make the best realtime decision and work with a 'well-oiled' multidisciplinary team with clear pathways for patients, good handoffs and communication practices $[8,9,37]$. Therefore, the operation room is an excellent milieu providing opportunities to teach not only technical skills but also nontechnical skills like clinical reasoning and teamwork.

Our profession is based on ethical behavior, which extends far beyond the rule of law [38]. Surgeons are often faced with the challenge of balancing truth-telling and the maintenance of hope in the setting of a poor prognosis or an adverse surgical event [25, 27]. Our study identified that both learners and surgical teachers agreed ethical issues were the learning preference for SL. It is necessary to understand how to communicate uncertainty and risk and how to make a disclosure to the family during or after an operation [27]. Several authors have suggested that training on disclosing adverse events using specific communication skills could be developed in the medical school curriculum $[35,39]$.

Several limitations in the present series has been identified. The current study recruited a relatively small number of learners and surgical teachers and examined practice at only one training center, so one could argue that it might not be reflective of all. Larger scale research on students and teacher perspectives at multimedical centers is necessary. Since both learners and surgical teachers have expectations mutually agreed on, this would optimize performance of every participant and reduce barriers of communication while increasing confidence, which would eventually lead to better learning outcome and patient safety [2].

\section{Conclusions}

Our study revealed significant disparity of perspectives between teachers and learners on perioperative teaching and learning. Resident perspective on learning objectives in the operating room differed between JL and SL even though both scrub in the same case, and there was discrepancy between resident's learning as perceived by teachers, particularly in junior learners. Surgical teachers should set an appropriate quantity and quality of learning objectives for different learner levels. Effective communication between teachers and learners has the potential to improve learning experience and create a positive environment in the operating room.

\section{Supplementary information}

Supplementary information accompanies this paper at https://doi.org/10. 1186/s12909-020-02172-8.

\section{Additional file 1.}

\section{Abbreviations}

PGY: Postgraduate year; JL: Junior learner; SL: Senior learner

\section{Acknowledgements}

The authors would like to acknowledge the Ministry of Health and Welfare in Taiwan for funding this project.

\section{Authors' contributions}

YTC: draft writing, PYL: revision, CSL: final decision, and all authors have read and approved the manuscript.

\section{Funding}

This work was supported by grants through funding from the Ministry of Health and Welfare in Taiwan. The role of the funding body was to build a consensus about learning and teaching objectives and improve the educational environment in the operating room.

Availability of data and materials

All data and materials are available.

Ethics approval and consent to participate

The study protocol was approved by the institutional review board of Kaohsiung Medical University Hospital (KMUHIRB-E(II)-20180262). All investigations were conducted according to the principles outlined in the Declaration of Helsinki.

Consent for publication

All authors authorized consent to publish.

\section{Competing interests}

No potential conflict of interest was reported by the authors.

\section{Author details}

${ }^{1}$ Department of Surgery, Kaohsiung Medical University Hospital, Kaohsiung, Taiwan. ${ }^{2}$ School of Medicine, College of Medicine, Kaohsiung Medical University, Kaohsiung, Taiwan. ${ }^{3}$ College of Humanities and Social Sciences, Kaohsiung Medical University, Kaohsiung, Taiwan. 
Received: 7 April 2020 Accepted: 22 July 2020

Published online: 31 July 2020

\section{References}

1. Smith T. Apprentice surgeons. BMJ. 1996;312:1233. https://doi.org/10.1136/ bmj.312.7040.1233

2. Kenton K. How to teach and evaluate learners in the operating room Obstet Gynecol Clin N Am. 2006;33:325-32, ix. https://doi.org/10.1016/j.ogc. 2006.02.003.

3. Drolet BC, Sangisetty S, Tracy TF, Cioffi WG. Surgical residents' perceptions of 2011 Accreditation Council for Graduate Medical Education duty hour regulations. JAMA Surg. 2013;148:427-33. https://doi.org/10.1001/jamasurg. 2013.169 .

4. Wang TH, Drolet BC, Tsai KY, Liu YF. Residents' perception of duty hour limits through teaching hospital accreditation status-experience in Taiwan. J Formos Med Assoc. 2017;116:398-401. https://doi.org/10.1016/j.jma.2016. 11.004.

5. The Ministry of Health and Welfare of Taiwan. Assessment of the workload of residents in Taiwan [Article in Chinese]. https://www.mohw.gov.tw/cp-2 736-8859-1.html. Assessed 31 Jan 2020.

6. Ministry of Labor: Medical Institution Agreement Stipulated by Article 84-1 of Labor Standards Act Section. https://law.moj.gov.tw/ENG/LawClass/ LawAll.aspx?pcode=N0030001. Assessed 31 Jan 2020.

7. Kim RH, Gilbert T, Ristig K, Chu QD. Surgical resident learning styles: faculty and resident accuracy at identification of preferences and impact on ABSITE scores. J Surg Res. 2013;184:31-6. https://doi.org/10.1016/j.jss.2013.04.050.

8. Lai CS, Lu PY, Chen TL. Teaching of clinical reasoning in surgery. Formosan J Med. 2016:20:290-6. https://doi.org/10.6320/FJM.2016.20(3).7.

9. Agha RA, Fowler AJ, Sevdalis N. The role of non-technical skills in surgery. Ann Med Surg (Lond). 2015;4:422-7. https://doi.org/10.1016/j.amsu.2015.10. 006.

10. Kieu V, Stroud L, Huang P, Smith M, Spychal R, Hunter-Smith D, Nestel D. The operating theatre as classroom: a qualitative study of learning and teaching surgical competencies. Educ Health (Abingdon). 2015;28:22-8. https://doi.org/10.4103/1357-6283.161845.

11. Iwaszkiewicz M, Darosa DA, Risucci DA. Efforts to enhance operating room teaching. J Surg Educ. 2008;65:436-40. https://doi.org/10.1016/j.jsurg.2008. 07.006 .

12. Timberlake MD, Mayo HG, Scott L, Weis J, Gardner AK. What do we know about intraoperative teaching? A systematic review. Ann Surg. 2017;266: 251-9. https://doi.org/10.1097/SLA.0000000000002131.

13. Snyder RA, Tarpley MJ, Tarpley JL, Davidson M, Brophy C, Dattilo JB. Teaching in the operating room: results of a national survey. J Surg Educ. 2012;69:643-9. https://doi.org/10.1016/j.jsurg.2012.06.007.

14. Vikis EA, Mihalynuk TV, Pratt DD, Sidhu RS. Teaching and learning in the operating room is a two-way street: resident perceptions. Am J Surg. 2008; 195:594-8. https://doi.org/10.1016/j.amjsurg.2008.01.004.

15. Roberts NK, Williams RG, Kim MJ, Dunnington GL. The briefing, intraoperative teaching, debriefing model for teaching in the operating room. J Am Coll Surg. 2009;208:299-303. https://doi.org/10.1016/j. jamcollsurg.2008.10.024.

16. Trehan A, Barnett-Vanes A, Carty MJ, McCulloch P, Maruthappu M. The impact of feedback of intraoperative technical performance in surgery: a systematic review. BMJ Open. 2015;5:e006759. https://doi.org/10.1136/ bmjopen-2014-006759.

17. Rose JS, Waibel BH, Schenarts PJ. Disparity between resident and faculty surgeons' perceptions of preoperative preparation, intraoperative teaching, and postoperative feedback. J Surg Educ. 2011;68:459-64. https://doi.org/10. 1016/j.jsurg.2011.04.003.

18. Butvidas LD, Anderson Cl, Balogh D, Basson MD. Disparities between resident and attending surgeon perceptions of intraoperative teaching. Am J Surg. 2011;201:385-9. https://doi.org/10.1016/j.amjsurg.2010.08.027.

19. Vollmer CM Jr, Newman LR, Huang G, Irish J, Hurst J, Horvath K. Perspectives on intraoperative teaching: divergence and convergence between learner and teacher. J Surg Educ. 2011;68:485-94. https://doi.org/10.1016/j.jsurg. 2011.05.010.

20. Levinson KL, Barlin JN, Altman K, Satin AJ. Disparity between resident and attending physician perceptions of intraoperative supervision and education. J Grad Med Educ. 2010;2:31-6. https://doi.org/10.4300/JGME-D09-00096.1.
21. Hsu PW, Hsieh MJ, Fu RH, Huang JL, Liao MC, Lee ST. Comparing the outcomes of different postgraduate year training programs in Taiwan. Biom J. 2015;38:544-9. https://doi.org/10.1016/j.bj.2016.01.006.

22. Beard J, Strachan A, Davies H, Patterson F, Stark P, Ball S, Taylor P, Thomas S. Developing an education and assessment framework for the Foundation Programme. Med Educ. 2005;39:841-51. https://doi.org/10.1111/j.1365-2929. 2005.02236.x.

23. Teo A. The current state of medical education in Japan: a system under reform. Med Educ. 2007;41:302-8. https://doi.org/10.1111/j.1365-2929.2007. 02691.x.

24. The American Broad of Surgery. Training \& certification. http://www. absurgery.org/default.jsp?certgsqe_resassess. Assessed 21 May 2020

25. Yule S, Flin R, Maran N, Rowley D, Youngson G, Paterson-Brown S. Surgeons' non-technical skills in the operating room: reliability testing of the NOTSS behavior rating system. World J Surg. 2008:32:548-56. https://doi.org/10. 1007/s00268-007-9320-z.

26. Lai CS, Huang SH, Shen CJ, Wang CN, Tsai JC, Yen JH. A practical way to enhance the intraoperative teaching and learning-anatomy-based clinical reasoning. Helsinki: Oral presentation at: The Association for Medical Education in Europe Annual Meeting; 2017.

27. Elwy AR, Itani KM, Bokhour BG, Mueller NM, Glickman ME, Zhao S, Rosen AK, Lynge D, Perkal M, Brotschi EA, Sanchez VM, Gallagher TH. Surgeons' disclosures of clinical adverse events. JAMA Surg. 2016;151:1015-21. https://doi.org/10.1001/jamasurg.2016.1787.

28. Hu YH, Wang CY, Huang MS, Lee CH, Wen YS. Analysis of the causes of surgery-related medical disputes in Taiwan: need for acute care surgeons to improve quality of care. J Chin Med Assoc. 2016;79:609-13. https://doi.org/ 10.1016/j.jcma.2016.04.008 Epub 2016 Aug 28.

29. Marshall DC, Salciccioli JD, Walton SJ, Pitkin J, Shalhoub J, Malietzis G. Medical student experience in surgery influences their career choices: a systematic review of the literature. J Surg Educ. 2015;72:438-45. https://doi.org/10.1016/j.jsurg.2014.10.018 Epub 2014 Dec 24

30. Statistics of licensed medical institutions of 2018 in Taiwan [Article in Chinese]. http://www.tma.tw/stats/index_AllPDF.asp. Accessed 31 Jan 2020.

31. Lawal TA, Afolabi AO. Factors influencing the choice of surgery as a career by pre-registration interns. Afr Health Sci. 2013;13:814-9. https://doi.org/10. 4314/ahs.v13i3.42

32. Assessment of the carrier development of the medical populations in Taiwan [Article in Chinese]. http://www.nhri.org.tw/NHRI_WEB/nhriw001 Action.do?status=Show_Dtl\&nid=20141219679218210000\&uid=20081204954 976470000. Accessed 31 Jan 2020.

33. Park J, Ponnala S, Fichtel E, Tehranchi K, Fitzgibbons S, Parker SH, Lau N, Safford SD. Improving the intraoperative educational experience: understanding the role of confidence in the resident-attending relationship. J Surg Educ. 2019;76(5):1187-99. https://doi.org/10.1016/j.jsurg.2019.02.012.

34. Torbeck L, Williams RG, Choi J, Schmitz CC, Chipman JG, Dunnington GL. How much guidance is given in the operating room? Factors influencing faculty self-reports, resident perceptions, and faculty/resident agreement. Surgery. 2014;156(4):797-803. https://doi.org/10.1016/j.surg.2014.06.069.

35. Whittaker G, Abboudi H, Khan MS, Dasgupta P, Ahmed K. Teamwork assessment tools in modern surgical practice: a systematic review. Surg Res Pract. 2015;2015:494827. https://doi.org/10.1155/2015/494827.

36. Wood TC, Raison N, Haldar S, Brunckhorst O, Mcllhenny C, Dasgupta P, Ahmed K. Training tools for nontechnical skills for surgeons-a systematic review. J Surg Educ. 2017;74:548-78. https://doi.org/10.1016/j.jsurg.2016.11.017.

37. Manser T, Foster S. Effective handover communication: an overview of research and improvement efforts. Best Pract Res Clin Anaesthesiol. 2011;25: 181-91. https://doi.org/10.1016/j.bpa.2011.02.006

38. Mavroudis C. Ethical forces that shape a career in surgery. Am J Surg. 2005; 190:319-23. https://doi.org/10.1016/j.amjsurg.2005.05.034.

39. Hagemann V, Herbstreit F, Kehren C, Chittamadathil J, Wolfertz S, Dirkmann D, Kluge A, Peters J. Does teaching non-technical skills to medical students improve those skills and simulated patient outcome? Int J Med Educ. 2017; 8:101-13. https://doi.org/10.5116/ijme.58c1.9f0d.

\section{Publisher's Note}

Springer Nature remains neutral with regard to jurisdictional claims in published maps and institutional affiliations. 